\title{
On the Effect of Through-Thickness Integration for the Blank Thickness and Ear Formation in Cup Drawing FE Analysis
}

\author{
Bora Şener ${ }^{1}(\mathbb{1})$, Toros Arda Akşen² ${ }^{2 *}$, Mehmet Fırat ${ }^{2}$ (i) \\ ${ }^{1}$ Yildiz Technical University, Faculty of Mechanical Engineering, Mechanical Engineering Department/Istanbul, Turkey \\ ${ }^{2}$ Sakarya University, Faculty of Engineering, Mechanical Engineering Department/Sakarya, Turkey
}

\begin{abstract}
Various numerical parameters such as element size, mesh topology, element formulations effect the prediction accuracy of sheet metal forming simulations and wrong selection of these parameters can lead to inaccurate predictions. Therefore, selection of proper numerical parameters is crucial for obtaining of realistic results from finite element (FE) analyses. In the present work, influence of the number of through-thickness integration points from the numerical parameters was investigated on the cup drawing simulation. Highly anisotropic AA 2090-T3 aluminum alloy was selected as test material and the anisotropic behavior of the material was defined with Barlat 91 yield criterion. Firstly, cup drawing model was created with implicit code Marc and then FE analyses were performed with five, seven and nine layers to investigate the effect of number of throughthickness integration points. The computed earing profiles and thickness strain distributions were compared with measurements. Comparisons showed that the angular locations of maximum cup heights and thickness strain distributions along rolling and transverse directions were captured accurately by Yld91 yield criterion and also it was observed that the layer number effects the maximum cup height and thickness strain distribution along the rolling direction.
\end{abstract}

Keywords: Cup Drawing, Finite Element Simulation, Earing Profile, Thickness Strain

\section{INTRODUCTION}

Governments force automotive companies to reduce exhaust emissions and improve fuel efficiency. In order to satisfy these requirements, engineers develop projects on reducing vehicle weight [1]. At this point, aluminum (Al) alloys become attractive for automotive manufacturers because of their lightweight and strength-to-weight ratios. Although $\mathrm{Al}$ alloys are preferred by most automotive manufacturers, modelling of these materials is difficult due to complex anisotropic behaviors. Anisotropic behavior of the sheet materials is represented by orthotropic anisotropic yield criteria. Various anisotropic yield criteria have been developed in the literature. The first anisotropic yield criterion was proposed by Hill in 1948 [2]. Hill48 criterion has a quadratic form and it gives consistent results for steels. However, conventional Hill's quadratic criterion can't represent the anisotropic behavior of aluminum alloys. According to Hillus yield criterion, biaxial yield stress ratio $\left(\sigma_{b} / \sigma_{0}\right)$ is lower than unity when the normal anisotropy coefficient $\left(r_{n}\right)$ is less than unity and also $r_{0}>r_{90}$ when $\sigma_{\mathrm{u}}>\sigma$. However, experimental studies performed by Woodthorpe and Pearce for some $\mathrm{Al}$ alloys showed that $\sigma_{b} / \sigma_{0}$ was always around 1.1 and $r_{n}$ varied between 0.5 to 0.6 and $r_{0}>r_{90}$ then $\sigma_{0}<\sigma_{90}$ [3]. This behavior was referred as "the first and the second anomalous" behavior by researchers. Therefore, non-quadratic yield functions are required to use for describing the anisotropic behavior of these materials. Hill developed a non-quadratic yield criterion in 1979 [4]. Hill79 criterion can model the first anomalous behavior, but it couldn't model second anomalous behavior of $\mathrm{Al}$ alloys. Then, Hill improved his yield criterion and proposed a new non-quadratic yield criterion in 1990 [5]. Hill90 criterion can describe both the first and second anomalous behaviors, but this model is not user friendly and it requires long simulation time for FE analysis. Unlike Hill's models, Barlat et al. developed various anisotropic yield functions for $\mathrm{Al}$ alloys that these functions are referred as Yld89 [6], Yld91 [7], Yld96 [8], Yld2000-2d [9] and Yld2004 [10] in the literature. These models are derived from linear transformation approach and give better results than Hill's models for Al-alloys.

Several studies have been carried out about the modeling of Al-alloys in the literature. Chung and Shah [11] defined the anisotropic behavior of AA 2008-T4 alloy using Barlat91 
(Yld91) yield criterion and performed FE analyses of the bulge and cup drawing tests. They compared the predicted bulge profiles, cup height and thickness values with experimental results. Similar studies were carried out by Yoon et al. [12] and Chung et al. [13]. They predicted earing profiles and thickness strain distributions with FE simulations and found good agreement between FE simulation and experiment. Then Yoon et al. used Yld91 yield criterion to predict earing of AA 2008-T4 alloy and compared with Hill48 [14]. Parente et al. modeled the cup drawing of AA 2008-T4 alloy with solid-shell elements and Yld91 yield criterion [15]. They applied the model to predict earing and obtained a good agreement with experimental results. Yoon et al. [16] applied Yld2004 yield criterion to predict earing of AA2090-T3 alloy. They used solid elements in FE analyses and succesfully predicted the earing profile of the cup. Younas et al. [17] investigated the effect of solid-shell elements on the earing prediction and compared with standard solid elements. They performed FE simulations of cup drawing tests with Hill48, Yld91 and Yld2004 yield functions for AA2090-T3 alloy and obtained closer results with non-quadratic yield functions to the experiments. Although succesfull results were obtained in these studies, it is seen that researchers haven't performed any study about the influence of number of through-thickness integration points in cup drawing simulations.

In this study, this effect was investigated for highly anisotropic AA2090-T3 aluminum alloy. Anisotropy of the material was defined with Yld91 yield criterion and FE analyses were carried out with different number of integration points. Then the computed earing profiles and thickness distributions were compared with measurements.

\section{MATERIAL AND METHOD}

\subsection{Plasticity Model}

A yield criterion, a flow rule and a hardening rule must be defined in order to establish a plasticity model. Yield criterion defines the elastic boundary in stress space, flow rule determines the direction of plastic strain increment and hardening rule defines the evolution of yield surface. In this study, Yld91 yield criterion was used for definition of initial anisotropy of the material and the criterion is explained in below:

Yld91 is a six-component yield criterion and it is developed by Barlat et al. to identify anisotropic behavior of Al-alloys. This criterion is based on the linear transformation approach and expressed as the following:

$$
\phi=\left|S_{1}-S_{2}\right|^{m}+\left|S_{2}-S_{3}\right|^{m}+\left|S_{3}-S_{1}\right|^{m}=2 \bar{\sigma}^{m}
$$

where $\bar{\sigma}$ is the effective stress, $S_{1}, S_{2}$ and $S_{3}$ are the principal values of the transformed deviatoric stress tensor $S_{\alpha \beta}$,

$$
S_{\alpha \beta}=\left[\begin{array}{ccc}
S_{x x} & S_{x y} & S_{x z} \\
S_{y x} & S_{y y} & S_{y z} \\
S_{z x} & S_{z y} & S_{z z}
\end{array}\right]=\left|\begin{array}{ccc}
\frac{C_{3} C-C_{2} B}{3} & C_{6} H & C_{5} G \\
C_{6} H & \frac{C_{1} A-C_{3} C}{3} & C_{4} F \\
C_{5} G & C_{4} F & \frac{C_{2} B-C_{1} A}{3}
\end{array}\right|
$$

here

$$
\begin{aligned}
& A=\sigma_{y y}-\sigma_{z z}, B=\sigma_{z z}-\sigma_{x x}, \quad C=\sigma_{x x}-\sigma_{y y}, \\
& F=\sigma_{y z}, \quad G=\sigma_{z x}, \quad H=\sigma_{x y}
\end{aligned}
$$

Linear transformation of the deviatoric symmetric stress tensor can be expressed as follows for plane stress conditions.

$$
S_{\alpha \beta}=\left[\begin{array}{ccc}
S_{x x} & S_{x y} & 0 \\
S_{y x} & S_{y y} & 0 \\
0 & 0 & S_{z z}
\end{array}\right]=\left|\begin{array}{ccc}
\frac{C_{3}\left(\sigma_{x x}-\sigma_{y y}\right)-C_{2} \sigma_{x x}}{3} & C_{6} \sigma_{x y} & 0 \\
C_{6} \sigma_{x y} & \frac{C_{1} \sigma_{y y}-C_{3}\left(\sigma_{x x}-\sigma_{y y}\right)}{3} & 0 \\
0 & 0 & -S_{x x}-S_{y y}
\end{array}\right| \text { (4) }
$$

where $C_{i=1 \sim 6}$ are the constants describing the anisotropy of the material. When $C_{i=1 \sim 6}=1$, the material is isotropic and the criterion transforms Tresca yield criterion for $m=1$ or $\infty$ and von Mises criterion for $m=2$ or 4 . The parameter $m$ is related to the crystallografic structure of the material. This parameter is taken as 6 for body-centered-cubic and 8 for face-centered-cubic materials. Also yield surface is convex for $m>1$. For the plane stress state, the number of constants reduces to four $\left(C_{1}, C_{2}, C_{3}\right.$ and $\left.C_{6}\right)$. These constants could be determined by solving the system of nonlinear equations [18]. Depending on the yield stresses along three directions $\left(0^{\circ}, 45^{\circ}\right.$ and $\left.90^{\circ}\right)$ and equibiaxial yield stress $\left(\sigma_{b}\right)$ these equations are written as following:

$$
\begin{aligned}
& \mathrm{f}_{1}\left(\mathrm{C}_{\mathrm{i}}\right)=\sigma_{\mathrm{equ}}\left(\sigma_{\mathrm{xx}}=\sigma_{\mathrm{b}}, \sigma_{y y}=\sigma_{\mathrm{b}}, \sigma_{\mathrm{xy}}=0\right)-\sigma_{\mathrm{b}}=0 \text { for } \mathrm{i}=1,2,3,6 \\
& \mathrm{f}_{2}\left(\mathrm{C}_{\mathrm{i}}\right)=\sigma_{\mathrm{eqy}}\left(\sigma_{\mathrm{xx}}=\sigma_{0}, \sigma_{y y}=0, \sigma_{\mathrm{xy}}=0\right)-\sigma_{0}=0 \text { for } \mathrm{i}=1,2,3,6 \\
& \mathrm{f}_{3}\left(\mathrm{C}_{\mathrm{i}}\right)=\sigma_{\mathrm{equ}}\left(\sigma_{\mathrm{xx}}=\frac{\sigma_{45}}{2}, \sigma_{y y}=\frac{\sigma_{45}}{2}, \sigma_{x y}=\frac{\sigma_{45}}{2}\right)-\sigma_{45}=0 \text { for } \mathrm{i}=1,2,3,6 \\
& \mathrm{f}_{4}\left(\mathrm{C}_{\mathrm{i}}\right)=\sigma_{\mathrm{eqy}}\left(\sigma_{\mathrm{xx}}=0, \sigma_{\mathrm{yy}}=0, \sigma_{\mathrm{xy}}=\sigma_{90}\right)-\sigma_{90}=0 \text { for } \mathrm{i}=1,2,3,6
\end{aligned}
$$

The Newton-Raphson (N-R) numerical method was used to solve the system of nonlinear equations in this study (Eg.58).

Associated flow rule was used to determine the plastic strain increments. It can be expressed as following equation:

$$
d \varepsilon_{i j}^{p}=d \lambda \frac{d \emptyset}{d \sigma_{i j}}
$$

where $d \varepsilon_{i j}^{p}$ shows the increment in the plastic strain tensor, $\mathrm{d} \lambda$ is the plastic multiplier, $\phi$ is the yield function and $\sigma_{\mathrm{ij}}$ denotes the Cauchy stress tensor. Isotropic hardening rule was assumed and hardening curve was defined with Swift power law is given in Eq. (10). 


$$
\sigma=K\left(\varepsilon_{0}+\varepsilon_{p}\right)^{n}
$$

where $K$ is the strength coefficient, $\varepsilon_{0}$ is initial plastic strain and $n$ is strain hardening coefficient. The parameters in Eq. (10) are given in Table 1.

Table 1. Swift parameters for AA2090-T3 [19]

\begin{tabular}{|c|c|c|}
\hline $\mathrm{K}$ & $\boldsymbol{\varepsilon}_{0}$ & $\mathrm{n}$ \\
\hline 646 & 0.025 & 0.227 \\
\hline
\end{tabular}

\subsection{Finite Element Method}

In the present work, cup drawing process of AA2090-T3 alloy was investigated. Information about tool geometry and dimensions can be found in the study [19].

The process was modelled by using Marc software. Full models of the parts were prepared to reflect anisotropy of the material. The blank was discretized by shell elements, full-integrated element formulation was selected and different number of through-thickness integration points were used to study the effect of the number of layers. Therefore, three different FE models were built and the blank was modeled with 5, 7 and 9 layers. FE model of the cup drawing process was shown in Figure 1. The tools were modeled as rigid bodies. The die was fixed in its initial position and the punch moved upwards according to position-time data table. $22.2 \mathrm{kN}$ blank holder force was applied. Segment to segment contact algorithm was used for defining the contact and the friction coefficient between the parts was taken as 0.1 .

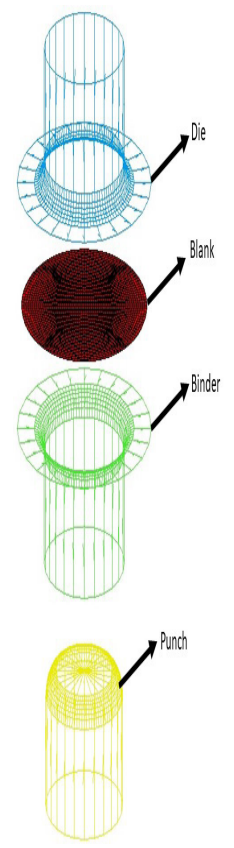

Figure 1. FE model of cylindrical cup deep drawing process

Required yield stress ratios for determination of Yld91 coefficients are given in Table 2 . In Table $2, \bar{\sigma}_{\theta}$ indicates the normalized yield stress along $\theta$ direction. In this study, yield stress in rolling direction $\left(\sigma_{0}\right)$ was taken as reference yield stress in the determination of yield stress ratios (Eq 11). Yld91 coefficients were determined by solving the system of nonlinear equations with N-R numerical method. Marc program takes yield stress ratios as input and calculates Yld91 coefficients with N-R numerical method. The determined Yld91 coefficients were given in Table 3.

$$
\bar{\sigma}_{\theta}=\frac{\sigma_{\theta}}{\sigma_{0}}
$$

Table 2. Yield stress ratios of AA2090-T3 in different directions and biaxial stress state [19]

\begin{tabular}{|c|c|c|c|c|}
\hline Material & $\overline{\boldsymbol{\sigma}}_{\mathbf{0}}$ & $\overline{\boldsymbol{\sigma}}_{\mathbf{4 5}}$ & $\overline{\boldsymbol{\sigma}}_{\mathbf{9 0}}$ & $\overline{\boldsymbol{\sigma}}_{\boldsymbol{b}}$ \\
\hline AA2090-T3 & 1.0000 & 0.8114 & 0.9102 & 1.0350 \\
\hline
\end{tabular}

Table 3. Anisotropy parameters of Yld91 yield criterion for AA2090-T3

\begin{tabular}{|c|c|c|c|}
\hline$C_{1}$ & $C_{2}$ & $C_{3}$ & $C_{6}$ \\
\hline 1.06746 & 0.855986 & 1.12964 & 1.29708 \\
\hline
\end{tabular}

\section{RESULTS AND DISCUSSION}

FE analyses were carried out with different number of layers. The predicted earing profiles and thickness strain distributions were compared with measurements to evaluate the influence of number of through-thickness integration points on the results. Experimental cup heights and thickness strain distributions were taken from literature [19].

\subsection{Comparison of Earing Profile}

In the present section, the influence of number of integration points on the earing prediction were examined. Therefore, the computed earing profiles from FE simulations were compared with measurements [19]. The final configuration of a full drawn cup and comparisons were shown in Figure 2 and Figure 3, respectively.

Figure 2. Equivalent stress distribution on fully drawn cup

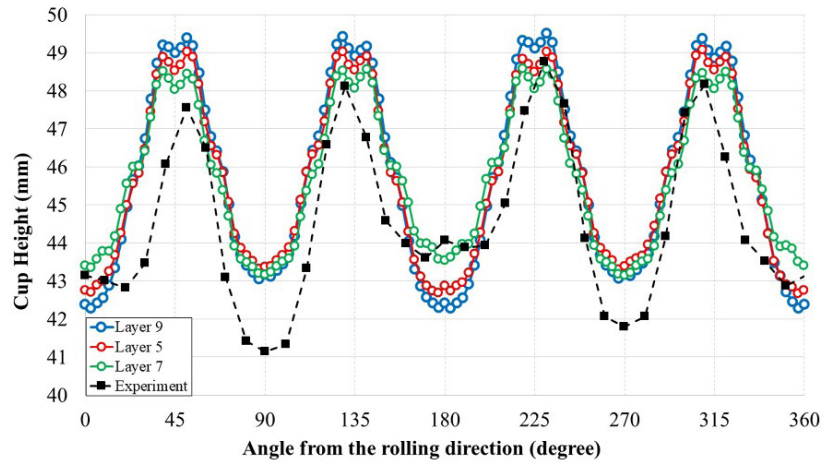

Figure 3. Computed and experimental cup heights [19]

It is seen from the Figure 3 that the earing profiles computed from simulations were similar and compatible with experiment, whereas the overall height predictions were different from each other. Cup height prediction of layer 7 was closer to the experiment than the other models at $135^{\circ}$ and $315^{\circ}$ from rolling direction, whereas the prediction of layer 5 was closer to the experimental data at $225^{\circ}$ from rolling. Besides, it was observed that the cup height predictions of the models at $90^{\circ}, 180^{\circ}$ and $270^{\circ}$ from rolling direction were incompatible with experimental results [19]. In addition to that it can be observed from the Figure 2 and 3 that four ears were predicted in the simulations, but six ears were observed in 
the experiment. Extra two ears were observed along the rolling directions in the experiment [19]. This is due to the coefficient identification procedure of Yld91 criterion. This criterion is a stress based yield criterion and only it takes only yield stress ratios as input.

\subsection{Comparison of Thickness Strains}

Thickness strains predicted from the analyses were compared with measurements [19]. Comparisons of the calculated and experimental thickness strains in rolling and transverse directions were shown in Figure 4 and Figure 5, respectively.

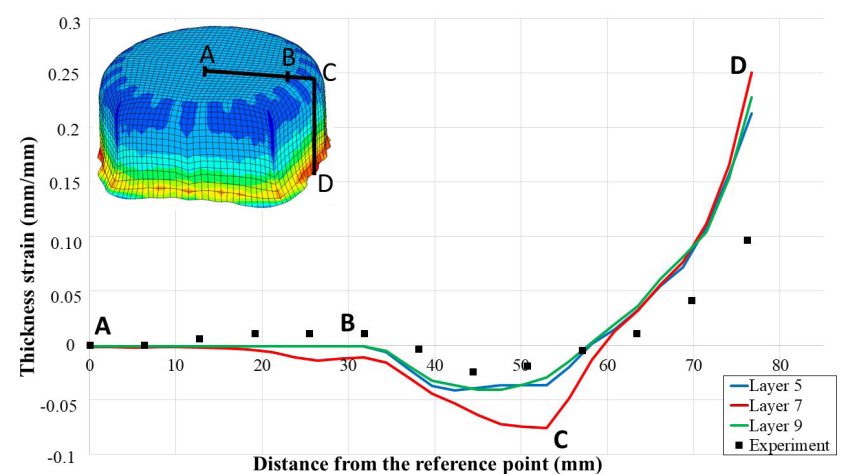

Figure 4. Computed and experimental thickness strains along the rolling direction [19]

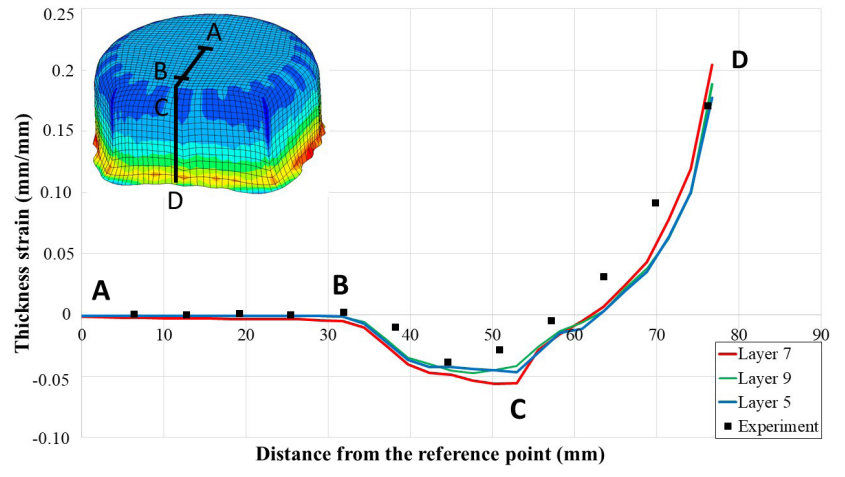

Figure 5. Computed and experimental thickness strains along the transverse direction [19]

It is seen from the Figure 4 and 5 that thickness strains along the rolling and transverse directions remained constant between reference point $\mathrm{A}$ and point $\mathrm{B}$, then they decreased until point $C$ and finally thickness strains contiously increased until point D. In both simulation and experiment, minimum and maximum thickness strains were observed on the corner region and the rim of the cup, respectively. This is an expected result, because both radial and tangential stresses occur on the corner region, whereas compressive stresses occur on the rim of the cup. Therefore, more thinning is observed on the corner region, while more thickening is observed on the rim of the part.

It is also could be observed from the Figure 4 and 5 that the differences of thickness strain between the FE simulation and experiment were small along rolling and transverse directions. Only, the predicted thickness strain from the layer 7 deviated from the experiment between 40 and $60 \mathrm{~mm}$ from the rolling direction.

\section{CONCLUSIONS}

Studies in the literature don't investigate the effect of through-thickness integration on the cup drawing simulations. In this study, the effect of this numerical parameter on the cup drawing simulation was studied. The anisotropic behavior of the material was defined with Yld91 yield criterion and implicit FE code Marc was used. Cup drawing simulations were performed with different layer numbers in order to investigate the effect of layer number through the thickness.

Conclusions drawn from this study are summarized in below:

1. Yld91 yield criterion could sufficiently define the anisotropic behavior of AA2090-T3 alloy

2. The computed earing profiles and thickness strain distributions from the simulations were compatible with the experiment.

3. Positioning of the ears on the drawn cup could be accurately predicted by Yld91 yield criterion. However differences between predicted and experimental cup heights were observed.

4. Layer number through the thickness from numerical parameters has a strong effect on the predicted maximum cup heights.

\section{REFERENCES}

[1] Miller, W.S., Zhuang, L., Bottema, J., Wittebrood, A.J., De Smet, P., Haszler, A., Vieregge, A. (2000). Recent development in aluminium alloys for the automotive industry. Materials Science and Engineering A, 280(1): 37-49. doi: 10.1016/S0921-5093(99)00653-X.

[2] Hill, R. (1948). A theory of the yielding and plastic flow of anisotropic metals. Proceedings of the Royal Society of London Series A Mathematical and Physical Sciences, 193: 281-297. doi: 10.1098/ rspa.1948.0045.

[3] Woodthorpe, J., Pearce, R. (1970). The anomalous behavior of aluminium sheet under balanced biaxial tension. International Journal of Mechanical Sciences, 12(4): 341-347. doi: 10.1016/00207403(70)90087-1.

[4] Hill, R. (1979). Theoretical plasticity of textured aggregates. Mathematical Proceedings of the Cambridge Philosophical Society, 85: 179-191. doi: 10.1017/S0305004100055596

[5] Hill, R. (1990). Constitutive modelling of orthotropic plasticity in sheet metals. Journal of the Mechanics and Physics of Solids, 38(3): 405-417. doi: 10.1016/0022-5096(90)90006-P.

[6] Barlat, F., Lian, K. (1989). Plastic behavior and stretchability of sheet metals. Part I: A yield function for orthotropic sheets under plane stress conditions. International Journal of Plasticity, 5(1): 51-66. doi: 10.1016/0749-6419(89)90019-3.

[7] Barlat, F., Lege, D.J., Brem, J.C. (1991). A six-component yield function for anisotropic materials. International Journal of Plasticity, 7(7): 693-712. doi: 10.1016/0749-6419(91)90052-Z.

[8] Barlat, F., Maeda, Y., Chung, K., Yanagawa, M., Brem, J.C., Hayashida, Y., Lege, D.J., Matsui, K., Murtha, S.J., Hattori, S., Becker, R.C., Makosey, S. (1997). Yield finction development for aluminum alloy sheets. Journal of the Mechanics and Physics of Solids, 45(11-12): 17271763. doi: 10.1016/S0022-5096(97)00034-3 
[9] Barlat, F., Brem, J.C., Yoon, J.W., Chung, K., Dick, R.E., Lege, D.J., Pourboghrat, F., Choi, S-H., Chu, E. (2003). Plane stress yield function for aluminum alloy sheets-part 1: theory. International Journal of Plasticity, 19: 1297-1319. doi: 10.1016/s0749-6419(02)00019-0.

[10] Barlat, F., Aretz, H., Yoon, J.W., Karabin, M.E., Brem, J.C., Dick, R.E. (2005). Linear transformation-based anisotropic yield functions. International Journal of Plasticity, 21: 1009-1039. doi: 10.1016/j.ijplas.2004.06.004.

[11] Chung, K., Shah, K. (1992). Finite element simulation of sheet metal forming for planar anisotropic metals. International Journal of Plasticity, 8(4): 453-476. doi: 10.1016/0749-6419(92)90059-L.

[12] Yoon, J.W., Song, I.S., Yang, D.Y., Chung, K., Barlat, F. (1995). Finite element method for sheet forming based on an anisotropic strainrate potential and the convected coordinate system. International Journal of Mechanical Sciences, 37(7): 733-752. doi: 10.1016/00207403(95)00003-G.

[13] Chung, K., Lee, S.Y., Barlat, F., Keum, Y.T., Park, J.M. (1996). Finite element simulation of sheet forming based on a planar anisotropic strain-rate potential. International Journal of Plasticity, 12(1), 93-115. doi: 10.1016/S0749-6419(95)00046-1.

[14] Yoon, J.W., Yang, D.Y., Chung, K. (1999). Elasto-plastic finite element method based on incremental deformation theory and continuum based shell elements for planar anisotropic sheet materials. Computer Methods in Applied Mechanics and Engineering, 174(1-2), 23-56. doi: 10.1016/S0045-7825(98)00275-8.

[15] Parente, M.P.L., Valente, R.A.F., Jorge, R.M.N., Cardoso, R.P.R., De Sousa, R.J.A. (2006). Sheet metal forming simulation using EAS solid-shell finite elements. Finite Elements in Analysis and Design, 42(13), 1137-1149. doi: 10.1016/j.finel.2006.04.005.

[16] Yoon, J.W., Barlat, F., Dick, R.E., Karabin, M.E. (2006). Prediction of six or eight ears in a drawn cup based on a new anisotropic yield function. International Journal of Plasticity, 22, 174-193. doi: 10.1016/j. ijplas.2005.03.013.

[17] Younas, N., Chalal, H., Abed-Meraim, F. (2020). Finite element simulation of sheet metal forming processes using non-quadratic anisotropic plasticity models and solid-shell finite elements. Procedia Manufacturing, 47, 1416-1423. doi: 10.1016/j.promfg.2020.04.302.

[18] Banabic, D. (2010). Sheet Metal Forming Processes Constitutive Modelling and Numerical Simulation. Springer-Verlag, Berlin.

[19] Yoon, J.W., Barlat, F., Chung, K., Pourboghrat, F., Yang, D.Y. (2000). Earing predictions based on asymmetric nonquadratic yield function. International Journal of Plasticity, 16(9), 1075-1104. doi: 10.1016/ S0749-6419(99)00086-8. 